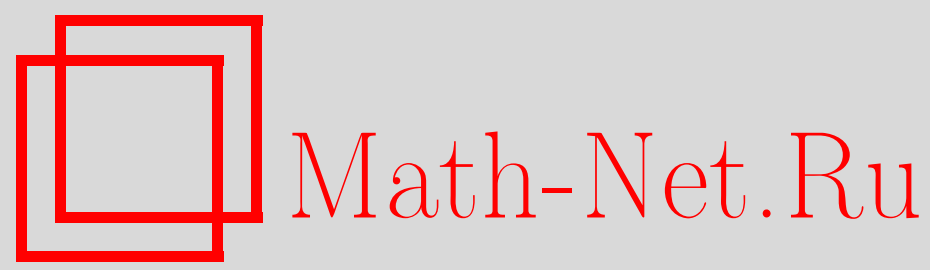

И. Б. Брыскин, Е. М. Семёнов, Недополняемые подпространства пространств Лоренца, Матем. заметки, 2000, том 68, выпуск 1, 57-65

DOI: https://doi.org/10.4213/mzm919

Использование Общероссийского математического портала Math-Net.Ru подразумевает, что вы прочитали и согласны с пользовательским соглашением http://www . mathnet.ru/rus/agreement

Параметры загрузки:

IP : 3.82 .47 .9

26 апреля 2023 г., $12: 21: 36$ 


\title{
НЕДОПОЛНЯЕМЫЕ ПОДПРОСТРАНСТВА ПРОСТРАНСТВ ЛОРЕНЦА
}

\author{
И. Б. Брыскин, Е. М. Семёнов
}

\begin{abstract}
В работе строится такая система ограниченных функций, что подпространство, порожденное этой системой, недополняемо в пространстве Лоренца $\Lambda(\alpha)$ для всех $\alpha \in$ $(0,1)$. Получены нижние оценки норм проектора на такие подпространства.

Библиография: 4 названия.
\end{abstract}

Если $1 \leqslant p<\infty$ и $\left\{x_{i}\right\}$ - система дизъюнктных функций (т.е. имеющая непересекающиеся носители) из $L_{p}[0,1]$, то $\overline{\operatorname{span}}\left(\left\{x_{i}\right\}, L_{p}\right)$ - подпространство $L_{p}$, порожденное системой $\left\{x_{i}\right\}$, дополняемо в $L_{p}$. Действительно, оператор

$$
P x(t)=\sum_{i=1}^{\infty} \int_{0}^{1} x(s) y_{i}(s) d s x_{i}(t)
$$

где $y_{i} \in L_{p^{\prime}}\left(1 / p+1 / p^{\prime}=1\right),\left\|y_{i}\right\|_{L_{p^{\prime}}}=1$ и

$$
\int_{0}^{1} x_{i}(s) y_{i}(s) d s=\left\|x_{i}\right\|_{L_{p}}
$$

имеет единичную норму в $L_{p}$ и проектирует на $\overline{\operatorname{span}}\left(\left\{x_{i}\right\}, L_{p}\right)$. Обратное утверждение для банаховых решеток доказал Т. Андо ([1] или $[2,1 . \mathrm{b} .8])$. Пусть $E$ - банахова решетка размерности больше 2. Если для любой замкнутой подрешетки $F \subset E$ сушествует положительньй оператор единичной нормы, проектирующий $E$ на $F$, то $E$ порядково изометрично $L_{p}(\mu)$ для некоторого $1 \leqslant p<\infty$ и некоторой меры $\mu$ или $c_{0}(\Gamma)$ для некоторого множества Г. Изоморфньй случай был рассмотрен Л. Цафрири, ([3] или [2, 1.b.12]). Пусть $E$ - порядково непрерьвная банахова решетка. Всякая подрешетка дополняема в $E$ тогда и только тогда, когда $E$ порядково изоморфна $L_{p}(\mu)$ для некоторого $p \in[1, \infty)$ и некоторой меры $\mu$ или $c_{0}(\Gamma)$ для некоторого множества $\Gamma$.

В настоящей работе строится такая система ограниченных дизъюнктных функций, что подпространство, порожденное этой системой, недополняемо в пространстве Лоренца $\Lambda(\alpha)$ для всех $\alpha \in(0,1)$. Кроме того, найдена нижняя оценка нормы проектора на подпространство, порожденное конечной системой дизъюнктных функций.

Работа второго автора выполнена при поддержке Российского фонда фундаментальных исследований, грант № 95-01-00135. 
Приведем необходимые определения. Пусть $0<\alpha \leqslant 1$. Через $\Lambda(\alpha)$ обозначается множество измеримых функций, для которых

$$
\|x\|_{\alpha}=\alpha \int_{0}^{\infty} x^{*}(t) t^{\alpha-1} d t<\infty,
$$

где $x^{*}(t)$ - перестановка функций $|x(t)|$ в убьвающем порядке. Очевидно,

$$
\left\|\chi_{e}\right\|_{\alpha}=(\operatorname{mes} e)^{\alpha},
$$

где $\chi_{e}(t)$ - характеристическая функция измеримого множества е. Норму оператора $A$, действующего в $\Lambda(\alpha)$, также будем обозначать $\|A\|_{\alpha}$. Если $\mathrm{A}$ - положительньй оператор в $\Lambda(\alpha)$, то

$$
\|A\|_{\alpha}=\sup _{\operatorname{mes} e>0} \frac{\left\|A \chi_{e}\right\|_{\alpha}}{(\operatorname{mes} e)^{\alpha}}
$$

(см. $[4,2.5 .2]$.

Пусть $a>0$. Пространство Лоренца функций, заданных на $[0, a]$, определяется точно так же и обозначается $\Lambda(\alpha)(0, a)$. Пространства $\Lambda(\alpha)(0, a)$ и $\Lambda(\alpha)(0,1)$ изометричны. Изометрия реализуется оператором $a^{\alpha} \sigma_{1 / a}$, где

$$
\sigma_{a} x(t)=x\left(\frac{t}{a}\right)
$$

По измеримой функции $x(t)$ построим ее функцию распределения

$$
n_{x}(\tau)=\operatorname{mes}\{t:|x(t)| \geqslant \tau\} .
$$

Тогда

$$
\|x\|_{\alpha}=\int_{0}^{\infty}\left(n_{x}(\tau)\right)^{\alpha} d \tau
$$

(см. $[4,2.5 .4])$. Если $\operatorname{mes}(\operatorname{supp} x) \leqslant 1$ и $0<\alpha<\beta \leqslant 1$, то $\|x\|_{\beta} \leqslant\|x\|_{\alpha}$.

Из (3) вытекает выпуклость функции $f(\alpha)=\ln \|x\|_{\alpha}$. Это означает, что

$$
\|x\|_{\beta} \leqslant\|x\|_{\alpha}^{(\gamma-\beta) /(\gamma-\alpha)}\|x\|_{\gamma}^{(\beta-\alpha) /(\gamma-\alpha)}
$$

для любых $0 \leqslant \alpha<\beta<\gamma \leqslant 1$ и $x \in \Lambda(\alpha) \cap \Lambda(\gamma)$.

Сопряженное к $\Lambda(\alpha)(0, a)$ пространство изометрически изоморфно пространству $M(\alpha)(0, a)$, где $0<a \leqslant \infty$ и

$$
\|x\|_{M(\alpha)}=\sup _{0<\tau<a} \frac{\int_{0}^{\tau} x^{*}(t) d t}{\tau^{\alpha}} .
$$

В частности, если $x \in \Lambda(\alpha), y \in M(\alpha)$, то $x y \in L_{1}$ и

$$
\|x y\|_{L_{1}} \leqslant\|x\|_{\Lambda(\alpha)}\|y\|_{M(\alpha)}
$$

Более подробно о пространствах Лоренща см $[4,2.5]$. 
Лемма 1. Пусть $0<\theta<1$. Существует такая возрастающая последовательность чисел $n_{i}, i=0,1, \ldots$, что

$$
\begin{array}{ll}
\text { 1) } & \sum_{i=1}^{m} n_{i} \geqslant m^{1-\theta} n_{m}, \quad m=1,2, \ldots, \\
\text { 2) } & \sum_{i=1}^{m} \frac{n_{i}-n_{i-1}}{n_{i}} \geqslant \frac{1}{2} m^{\theta}, \quad m=1,2, \ldots, \\
\text { 3) } & \lim _{m \rightarrow \infty} \frac{n_{m+1}-n_{m}}{\ln n_{m+1}-\ln n_{m}}=\infty .
\end{array}
$$

ДоКАЗАТЕЛЬСТВО. Положим $n_{0}=1$. Если $n_{0}, n_{1}, \ldots, n_{m}$ уже определены, то $n_{m+1}$ можно выбрать так, чтобы

$$
n_{m+1} \geqslant(m+1)^{1-\theta} n_{m+1}-m^{1-\theta} n_{m}, \quad \frac{n_{m+1}-n_{m}}{n_{m+1}} \geqslant(m+1)^{\theta}-m^{\theta} .
$$

Действительно, эти неравенства эквивалентны следующим

$$
\frac{n_{m}}{m^{\theta}+1-(m+1)^{\theta}} \leqslant n_{m+1} \leqslant \frac{m^{1-\theta} n_{m}}{(m+1)^{1-\theta}-1} .
$$

Неравенство

$$
\frac{(m+1)^{1-\theta}-1}{m^{1-\theta}} \leqslant m^{\theta}+1-(m+1)^{\theta}
$$

имеет место при всех $m=1,2, \ldots$, так как

$$
\frac{(m+1)^{1-\theta}-1}{m^{1-\theta}}<1+\frac{1-\theta}{m}-\frac{1}{m^{1-\theta}}<1-\theta m^{\theta-1}<m^{\theta}+1-(m+1)^{\theta} .
$$

Потому если мы положим

$$
n_{m+1}=\frac{m^{1-\theta}}{(m+1)^{1-\theta}-1} n_{m}
$$

то $n_{m+1}>n_{m}$ и будет выполнены (5) и (6). Так как

$$
\frac{n_{m+1}-n_{m}}{\ln n_{m+1}-\ln n_{m}}=\frac{\left(m^{1-\theta}+1-(m+1)^{1-\theta}\right) n_{m}}{\left((m+1)^{1-\theta}-1\right) \ln \frac{m^{1-\theta}}{(m+1)^{1-\theta}-1}}
$$

и

$$
\ln \frac{m^{1-\theta}}{(m+1)^{1-\theta}-1}=\ln \left(1+\frac{m^{1-\theta}+1-(m+1)^{1-\theta}}{(m+1)^{1-\theta}-1}\right)<\frac{m^{1-\theta}+1-(m+1)^{1-\theta}}{(m+1)^{1-\theta}-1}
$$

TO

$$
\frac{n_{m+1}-n_{m}}{\ln n_{m+1}-\ln n_{m}}>n_{m}
$$


Левая часть (8) и равенство

$$
\prod_{m=1}^{\infty}\left(m^{\theta}+1-(m+1)^{\theta}\right)=0
$$

показывают, что $n_{m} \rightarrow \infty$. Отсюда и из (10) вытекает $(7)$.

По заданному $\alpha \in(0,1)$ построим последовательность функций

$$
x_{n}(t)=x_{n, \alpha}(t)=\frac{1}{n}\left(\max \left(t, 2^{-n}\right)\right)^{-\alpha} .
$$

Имеем

$$
\left\|x_{n}\right\|_{\alpha}=\frac{1}{n} \alpha \int_{0}^{1}\left(\max \left(t, 2^{-n}\right)\right)^{-\alpha} t^{\alpha-1} d t=\frac{1}{n}\left(1+\alpha \int_{2^{-n}}^{1} \frac{d t}{t}\right)=\frac{1+\alpha n \ln 2}{n} \leqslant 2
$$

и

$$
\operatorname{mes}\left\{t: x_{n}(t) \geqslant \tau\right\}=(n \tau)^{-1 / \alpha}, \quad \frac{2^{\alpha}}{n} \leqslant \tau \leqslant \frac{2^{n \alpha}}{n},
$$

для всех $n=1,2, \ldots$ По заданной последовательности возрастающих чисел $\left\{n_{i}\right\}$ построим последовательность дизъюнктных функций

$$
y_{n_{i}}(t)= \begin{cases}x_{n_{i}}(t-i+1), & i-1<t<i, \\ 0 & \text { для остальных } t,\end{cases}
$$

где $x_{n}(t)$ определены по формуле (11).

Лемма 2. Пусть $0<\alpha<1,1=n_{0}<n_{1}<\cdots<n_{m}$. Тогда

$$
\left\|\sum_{i=1}^{m} y_{n_{i}}\right\|_{\alpha} \geqslant \frac{\alpha}{3} \ln 2 \sum_{i=1}^{m} \frac{n_{i}-n_{i-1}}{n_{i}}
$$

ДокАЗАТЕЛЬСТво. Предположим дополнительно, что последовательность $\left(2^{n_{i} \alpha}\right) \times$ $1 / n_{i}$ монотонно возрастает по $i$. Тогда в силу (12) для любого $\tau>0$ имеем

$$
\begin{aligned}
& \operatorname{mes}\left\{t: \sum_{i=1}^{m} y_{n_{i}}(t)>\tau\right\}=\sum_{i=1}^{m} \operatorname{mes}\left\{t: x_{n_{i}}(t)>\tau\right\} \\
& \geqslant \sum_{i=1}^{m}\left(n_{i} \tau\right)^{-1 / \alpha} \chi_{\left.\left(\left(2^{\alpha}\right) / n_{i},\left(2^{\alpha}\right) / n_{i-1}\right)\right) \cup\left(\left(2^{n_{i-1} \alpha}\right) / n_{i-1},\left(2^{n_{i} \alpha}\right) / n_{i}\right)}(\tau) .
\end{aligned}
$$

Используя (3), получаем

$$
\begin{aligned}
\left\|\sum_{i=1}^{m} y_{n_{i}}\right\|_{\alpha} & =\int_{0}^{\infty}\left(\operatorname{mes}\left\{t: \sum_{i=1}^{m} y_{n_{i}}(t)>\tau\right\}\right)^{\alpha} d \tau \\
& \geqslant \sum_{i=1}^{m}\left(\int_{\left(2^{\alpha}\right) / n_{i}}^{\left(2^{\alpha}\right) / n_{i-1}}+\int_{\left(2^{n_{i-1} \alpha}\right) / n_{i-1}}^{\left(2^{n^{\alpha}}\right) / n_{i}}\right)\left(n_{i} \tau\right)^{-1} d \tau \\
& =\sum_{i=1}^{m} \frac{1}{n_{i}}\left(\ln \frac{n_{i}}{n_{i-1}}+\ln \frac{2^{n_{i} \alpha} n_{i-1}}{n_{i} 2^{n_{i-1}}}\right)=\alpha \ln 2 \sum_{i=1}^{m} \frac{n_{i}-n_{i-1}}{n_{i}} .
\end{aligned}
$$


Если же $\left(2^{n_{i} \alpha}\right) / n_{i}$ монотонно убьвает по $i$, то, повторяя предыдущие оценки, имеем

$$
\begin{aligned}
\left\|\sum_{i=1}^{m} y_{n_{i}}\right\|_{\alpha} & \geqslant \sum_{i=1}^{m} \frac{1}{n_{i}}\left(\ln \frac{n_{i}}{n_{i-1}}-\ln \frac{2^{n_{i} \alpha} n_{i-1}}{n_{i} 2^{n_{i-1} \alpha}}\right) \\
& \geqslant \sum_{i=1}^{m} \frac{1}{n_{i}}\left(\ln \frac{n_{i}}{n_{i-1}}+\ln \frac{2^{n_{i} \alpha} n_{i-1}}{n_{i} 2^{n_{i-1} \alpha}}\right)=\alpha \ln 2 \sum_{i=1}^{m} \frac{n_{i}-n_{i-1}}{n_{i}} .
\end{aligned}
$$

Для любого $j \in\{1,2, \ldots, m\}$ справедлива оценка

$$
\left\|\sum_{i=1}^{m} y_{n_{i}}\right\|_{\alpha} \geqslant \int_{\left(2^{\alpha}\right) / n_{j}}^{\left(2^{n_{j} \alpha}\right) / n_{j}}\left(n_{j} \tau\right)^{-1} d \tau=\frac{\alpha \ln 2\left(n_{j}-1\right)}{n_{j}} \geqslant \alpha \ln 2 \frac{n_{j}-n_{j-1}}{n_{j}} .
$$

Функция

$$
f(t)=\frac{2^{t \alpha}}{t}
$$

монотонно убывает на интервале $(0,1 /(\alpha \ln 2))$ и возрастает на интервале $(1 /(\alpha \ln 2), \infty)$. Поэтому найдется такой индекс $j$, что последовательность $\left(2^{n_{i} \alpha}\right) / n_{i}$ убьвает при $1 \leqslant$ $i \leqslant j$ и возрастает при $j+1 \leqslant i \leqslant m$. В силу (15) и (16)

$$
\left\|\sum_{i=1}^{m} y_{n_{i}}\right\|_{\alpha} \geqslant \alpha \ln 2 \sum_{i=1}^{j} \frac{n_{i}-n_{i-1}}{n_{i}}, \quad\left\|\sum_{i=1}^{m} y_{n_{i}}\right\|_{\alpha} \geqslant \alpha \ln 2 \sum_{i=j+1}^{m} \frac{n_{i}-n_{i-1}}{n_{i}} .
$$

Из полученных неравенств и (17) вытекает (14).

Нетрудно посчитать, что

$$
\lim _{n \rightarrow \infty}\left\|y_{n}\right\|_{\alpha}=\alpha \ln 2 .
$$

Это говорит о точности оценки (14).

Пусть теперь $\left\{n_{i}\right\}$ - последовательность из леммы 1 и $\theta=(1+\alpha) / 2$. В силу $(14),(16)$ и (5)

$$
\left\|\sum_{i=1}^{m} y_{n_{i}}\right\|_{\alpha} \geqslant \frac{\alpha \ln 2}{6} m^{(1+\alpha) / 2}
$$

и

$$
\frac{1}{n_{m}} \sum_{i=1}^{m} \frac{n_{i}-n_{i-1}}{n_{i}} \geqslant m^{(1-\alpha) / 2}
$$

Из (9) вытекает, что для всех $i \geqslant 1$

$$
n_{i} \geqslant n_{1}=\frac{n_{0}}{2^{1-\theta}-1}=\frac{1}{2^{(1-\alpha) / 2}-1} \geqslant \frac{1}{\sqrt{2}-1}=\sqrt{2}+1>2 .
$$

Обозначим

$$
B_{\alpha, m}=\operatorname{span}\left\{y_{n_{i}}, 1 \leqslant i \leqslant m\right\} .
$$


ЛЕмма 3. Пусть $0<\alpha<1, m$ - натуральное число и $P$ - проектор $\Lambda(\alpha)(0, m)$ на $B_{\alpha, m}$. Существует такое $C(\alpha)>0$, что

$$
\|P\|_{\alpha} \geqslant C(\alpha) m^{(1-\alpha) / 2}
$$

и $C(\alpha)$ возрастает на $(0,1)$.

ДоказАтельство. Так как оператор $P$ является конечномерным и $\Lambda^{*}(\alpha)=M(\alpha)$, TO

$$
P x(t)=\sum_{j=1}^{m} \int_{j-1}^{j} f_{j}(s) x(s) d s y_{n_{j}}(t),
$$

где $f_{j} \in M(\alpha)$,

$$
\int_{j-1}^{j} f_{j}(s) y_{n_{j}}(s) d s=1
$$

для всех $j=1,2, \ldots, m$. Положим

$$
f_{k j}=\left\{\begin{array}{l}
0, \text { если } \int_{j-1+2^{-k}}^{j-1+2^{-k+1}} f_{j}(t)(t-j+1)^{-\alpha} d t<1 / 2, \\
\int_{j-1+2^{-k}}^{j-1+2^{-k+1}} f_{j}(t)(t-j+1)^{-\alpha} d t \text { в противном случае }
\end{array}\right.
$$

для $j=1,2, \ldots, k=1,2, \ldots,\left[n_{j}\right]$. Из определений $(11),(13)$ и $(20)$ вытекает

$$
\begin{aligned}
n_{j} & \leqslant 2^{n_{j} \alpha} \int_{j-1}^{j-1+2^{-\left[n_{j}\right]}} f_{j}(s) d s+\sum_{k=1}^{\left[n_{j}\right]} \int_{j-1+2^{-k}}^{j-1+2^{-k+1}} f_{j}(s)(s-j+1)^{-\alpha} d s \\
& \leqslant 2+\sum_{k=1}^{\left[n_{j}\right]} f_{k j}+\frac{\left[n_{j}\right]}{2} .
\end{aligned}
$$

Для $j \geqslant 4$ отсюда получаем

$$
\sum_{k=1}^{\left[n_{j}\right]} f_{k j} \geqslant \frac{n_{j}}{2}-2 \geqslant \frac{n_{j}}{4}
$$

Если $f_{k j} \neq 0$, то

$$
\frac{1}{2} \leqslant f_{k j} \leqslant\|P\|_{\alpha} .
$$

Для заданного $1 \leqslant k \leqslant n_{m}$ обозначим $d_{k}=\min \left\{j: n_{j} \geqslant k\right\}$. Тогда

$$
\frac{1}{4} \sum_{j=1}^{m} n_{j} \leqslant \sum_{j=1}^{m} \sum_{k=1}^{\left[n_{j}\right]} f_{k j}=\sum_{k=1}^{\left[n_{m}\right]} \sum_{j=d_{k}}^{\left[n_{m}\right]} f_{k j}
$$

Найдем такой индекс $l \in\left\{1,2, \ldots, n_{m}\right\}$, что

$$
\frac{1}{4 n_{m}} \sum_{j=1}^{m} n_{j} \leqslant \sum_{j=d_{l}}^{\left[n_{m}\right]} f_{l j}
$$


и обозначим через $Q=\left\{j_{1}, j_{2}, \ldots, j_{r}\right\}$ множество $\left\{j: d_{l} \leqslant j \leqslant\left[n_{m}\right], f_{l j} \neq 0\right\}$. Тогда $1 / 2 \leqslant f_{l j} \leqslant\|P\|_{\alpha}$ для $j \in Q$ и

$$
\frac{1}{4 n_{m}} \sum_{j=1}^{m} n_{j} \leqslant\|P\|_{\alpha} r
$$

Рассмотрим функцию $z(t)=2^{l \alpha} \sum_{j \in Q} \chi_{\left(j-1, j-1+2^{-l}\right)}(t)$. Очевидно, $\|z\|_{\alpha}=r^{\alpha}$ и

$$
P z(t)=\sum_{j \in Q} 2^{l \alpha} \int_{j-1+2^{-l}}^{j-1+2^{-l+1}} f_{j}(s) d s y_{n_{j}}(t)
$$

Tak кaK

$$
2^{l \alpha} \int_{j-1+2^{-l}}^{j-1+2^{-l+1}} f_{j}(s) d s \geqslant \int_{j-1+2^{-l}}^{j-1+2^{-l+1}} f_{j}(s)(s-j+1)^{-\alpha} d s=f_{l j}
$$

в силу (18)

$$
\|P z\|_{\alpha} \geqslant \frac{1}{2}\left\|\sum_{j \in Q} y_{n_{j}}\right\|_{\alpha} \geqslant \frac{1}{12} \alpha \ln 2 r^{(1+\alpha) / 2} .
$$

Отсюда

$$
\|P\|_{\alpha} \geqslant \frac{\|P z\|_{\alpha}}{\|z\|_{\alpha}} \geqslant \frac{1}{12} \alpha \ln 2 r^{(1-\alpha) / 2} .
$$

Продолжая оценку с помощью (22), получаем

$$
\|P\|_{\alpha} \geqslant \frac{1}{12} \alpha \ln 2\left(\frac{1}{4\|P\|_{\alpha} n_{m}} \sum_{i=1}^{m} n_{m}\right)^{(1-\alpha) / 2}
$$

В силу (19)

$$
\|P\|_{\alpha} \geqslant\left(\frac{1}{48} \alpha \ln 2 m^{(1-\alpha) / 2}\right)^{2 / 3} .
$$

Этим установлено $(21)$ с $C(\alpha)=(\alpha \ln 2 / 48)^{2 / 3}$.

Полученная оценка может быть усилена. Если $0<\delta<1 / 2$, то существует константа $D(m, \delta)$ такая, что

$$
\|y\|_{L_{\infty}} \leqslant D(m, \delta)\|y\|_{\alpha}
$$

для всех $y \in B_{\alpha, m}$ и всех $\alpha \in[\delta, 1-\delta]$. Это непосредственно вытекает из $(9),(11)$. Как отмечалось ранее, пространства $\Lambda(\alpha)(0, m)$ и $\Lambda(\alpha)(0,1)$ изометричны. Обозначим через $B_{\alpha, m}^{0}$ образ $B_{\alpha, m}$ относительно канонической изометрии $m^{\alpha} \sigma_{1 / m}$. Переобозначая константу в (23), можно считать, что (23) вьполнено для всех $y \in B_{\alpha, m}^{0}$.

Пусть $0<\delta \leqslant \alpha<\beta \leqslant 1-\delta, m$ - натуральное число и $P$ - проектор $\Lambda(\alpha)$ на $B_{\alpha, m}^{0}$. В силу (4) и (23)

$$
\|P x\|_{\alpha} \leqslant\|P x\|_{L_{\infty}}^{1-\alpha / \beta}\|P x\|_{\beta}^{\alpha / \beta} \leqslant\left(D(m, \delta)\|P\|_{\alpha}\|x\|_{\alpha}\right)^{1-\alpha / \beta}\|P x\|_{\beta}^{\alpha / \beta}
$$


для любых $x \in \Lambda(\alpha)$. Отсюда

$$
\|P x\|_{\beta} \geqslant\|P x\|_{\alpha}^{\beta / \alpha}\left(D(m, \delta)\|P\|_{\alpha}\|x\|_{\alpha}\right)^{1-\beta / \alpha}
$$

и

$$
\begin{aligned}
\sup _{x \in \Lambda(\alpha)} \frac{\|P x\|_{\beta}}{\|x\|_{\alpha}} & \geqslant \sup _{x \in \Lambda(\alpha)}\left(\frac{\|P x\|_{\alpha}}{\|x\|_{\alpha}}\right)^{\beta / \alpha}\left(D(m, \delta)\|P\|_{\alpha}\right)^{1-\beta / \alpha} \\
& =\|P\|_{\alpha}^{\beta / \alpha}\left(D(m, \delta)\|P\|_{\alpha}\right)^{1-\beta / \alpha}=D^{1-\beta / \alpha}(m, \delta)\|P\|_{\alpha}
\end{aligned}
$$

Если дополнительно потребовать, чтобы

$$
\beta<\alpha\left(1+\ln ^{-1} D(m, \delta)\right)
$$

то

$$
\sup _{x \in \Lambda(\alpha)} \frac{\|P x\|_{\beta}}{\|x\|_{\alpha}} \geqslant \frac{1}{e}\|P\|_{\alpha} .
$$

ТЕОрема 1. Существует такая система ограниченных дизбюнктных функиий $Y$ на $[0, \infty]$, что подпространство $\overline{\operatorname{span}}(Y, \Lambda(\alpha))$ недополняемо в $\Lambda(\alpha)$ для всех $\alpha \in(0,1)$.

ДокАЗАтЕЛЬСтво. Пусть $j$ - натуральное число $\geqslant 4$. Константа $C(\alpha)$ из леммы 3 монотонно возрастает. Поэтому $C(\alpha) \geqslant C(1 / j)$ для $\alpha \in[1 / j, 1)$. Найдем такое натуральное $m$, что

$$
C\left(\frac{1}{j}\right) m^{1 /(3 j)} \geqslant j .
$$

Если $1 / j \leqslant \alpha \leqslant 1-1 / j$ и $P$ есть проектор $\Lambda(\alpha)$ на $B_{\alpha, m}$, то в силу леммы 3 и $(26)$

$$
\|P\|_{\alpha} \geqslant j
$$

Построим такую последовательность $0<\alpha_{1}<\cdots<\alpha_{q}<1$, что $\alpha_{1}=1 / j$, $\alpha_{q}=1-1 / j$ и

$$
\alpha_{k+2}<\alpha_{k}\left(1+\ln ^{-1} D\left(m, \frac{1}{j}\right)\right) .
$$

Обозначим через $Y_{j k}(j=4,5, \ldots, k=1,2, \ldots, q=q(j))$ образ системы $\left\{y_{n_{i}}, 1 \leqslant\right.$ $i \leqslant m\}$, построенной по $\alpha=\alpha_{k}$, при изометрии $m^{\alpha} \sigma_{1 / m}$ и последующем сдвиге так, чтобы функции из различных семейств имели непересекающиеся носители. Заметим, если $y \in \operatorname{span} Y_{j k}$, то mes $(\operatorname{supp} y) \leqslant 1$. Поэтому

$$
\|y\|_{\alpha_{k}} \geqslant\|y\|_{\alpha} \geqslant\|y\|_{\alpha_{k+2}}
$$

при $\alpha_{k}<\alpha<\alpha_{k+2}$.

Докажем, что система

$$
Y=\bigcup_{j=4}^{\infty} \bigcup_{k=1}^{q_{j}} Y_{j k}
$$


обладает требуемыми свойствами. Действительно, для любого $\alpha \in(0,1)$ можно найти такое $j \geqslant 4$, что $\alpha \in(1 / j, 1-1 / j)$. Затем найдем такое $1 \leqslant k \leqslant q-2$, что $\alpha_{k}<\alpha<\alpha_{k+2}$. Если $P$ есть проектор $\Lambda(\alpha)$ на $Y$, то в силу $(25),(27)$ и $(28)$

$$
\|P\|_{\alpha} \geqslant \sup _{x \in \Lambda\left(\alpha_{k}\right)} \frac{\|P x\|_{\Lambda\left(\alpha_{k+2}\right)}}{\|x\|_{\Lambda\left(\alpha_{k}\right)}} \geqslant \frac{1}{e}\|P\|_{\alpha_{k}} \geqslant \frac{j}{e} .
$$

Число $j$ можно было выбирать сколь угодно большим. Это доказьвает недополняемость $\overline{\operatorname{span}}(Y, \Lambda(\alpha))$ в $\Lambda(\alpha)$.

Отметим результаты противоположного характера. Если $e_{1}, e_{2}, \ldots$ - последовательность дизъюнктных подмножеств, то подпространство, порожденное их характеристическими функциями $\chi_{e_{1}}, \chi_{e_{2}}, \ldots$, дополняемо в любом перестановочно-инвариантном пространстве, в частности, в $\Lambda(\alpha)$ при всех $\alpha \in(0,1]$ и норма соответствующего проектора равна 1. Это вытекает из теоремы 2.а.4 из [2].

Так как $\Lambda(1)=L_{1}$, теорема 1 теряет силу при $\alpha=1$.

Авторы искренне признательны рецензенту за ценные замечания.

\section{СПИСОК ЦИТИРОВАННОЙ ЛИТЕРАТУРЫ}

[1] Ando T. Banachverbände und positive Projektionen // Math. Z. 1969. V. 109. P. 121-130.

[2] Lindenstrauss J., Tzafriri L. Classical Banach Spaces. II. Function Spaces. Berlin: Springer, 1979.

[3] Tzafriri L. An isomorphic characterization of $L_{p}$ and $c_{0}$ spaces. II // Michigan Math. J. 1971. V. 18. P. 21-31.

[4] Крейн С. Г., Петунин Ю. И., Семёнов Е. М. Интерполяция линейных операторов. М.: Наука, 1978.

(И.Б. Брыскин) Центр технологического образования 